\title{
Can Contrast-Enhanced Harmonic Endoscopic Ultrasonography Differentiate Malignancy from Benign Disease?
}

\author{
Tae Hoon Lee \\ Division of Gastroenterology, Department of Internal medicine, Soonchunhyang University College of Medicine, Cheonan Hospital, Cheonan, \\ Korea
}

See "Clinical Value of Contrast-Enhanced Harmonic Endoscopic Ultrasonography in the Differential Diagnosis of Pancreatic and Gallbladder Masses” by Galam Leem, Moon Jae Chung, Jeong Youp Park, et al., on page 80-88.

Endoscopic ultrasonography (EUS) was developed in the 1980 s and is now commonly used for diagnosis and treatment of pancreatobiliary disease. Diagnostic and interventional EUS techniques are diverse and include the detection of small pancreatic or biliary lesions, tumor staging, tumor and cyst ablation, and biliary drainage. However, conventional diagnostic EUS has several limitations. First, the sensitivity and specificity of diagnostic EUS relies on the experience of the endoscopist. Second, EUS cannot be used to assess tumor vascularity and hemodynamics. Third, the use of imaging techniques alone has not proven to be an adequate replacement for pathologic diagnosis. Finally, recent advances in computed tomography (CT) and magnetic resonance imaging (MRI) techniques have allowed for the collection of more objective and comprehensive data than was previously possible. Addressing some of the limitations of EUS, newer techniques have been developed that improve characterization of detected lesions. One of these techniques, EUS-guided fine needle aspiration or biopsy (EUS-FNA/B), represents a major advancement in the diagnosis of pancreatic tumors. To improve

Received: January 2, 2018 Revised: January 10, 2018

Accepted: January 11, 2018

Correspondence: Tae Hoon Lee

Division of Gastroenterology, Department of Internal Medicine, Soonchunhyang University College of Medicine, Cheonan Hospital, 31 Suncheonhyang 6-gil, Dongnam-gu, Cheonan 31151, Korea

Tel: +82-41-570-3662, Fax: +82-41-574-5762, E-mail: thlee9@schmc.ac.kr

(cc) This is an Open Access article distributed under the terms of the Creative Commons Attribution Non-Commercial License (http://creativecommons.org/ licenses/by-nc/3.0) which permits unrestricted non-commercial use, distribution, and reproduction in any medium, provided the original work is properly cited. the histological yield, novel EUS-FNA needles with core or sharp tips have recently been developed. However, despite EUS-FNA/B having a cytological and histological diagnostic accuracy as high as $95 \%$ for pancreatic masses, there is still a need for simpler and less invasive methods. ${ }^{1-5}$

Contrast-enhanced harmonic ultrasonography (CH-EUS) is a new emerging diagnostic technology that can be used to assess blood flow inside tissues. CH-EUS may improve the detectability of tumors, and allow characterization of tumor vascularity. Whereas a Doppler image can detect large vascular lesions, $\mathrm{CH}$-EUS takes advantage of the high image resolution of EUS and identifies smaller vessels by using microbubbles. It is also able to selectively depict microbubble signals from ultrasound contrast agents. ${ }^{1-4}$ Thus, $\mathrm{CH}-\mathrm{EUS}$ may be able to provide real-time information about a tumor, as compared to Doppler, CT, or MRI. Recently developed techniques for EUS system include tissue harmonic echo, elastography, and contrast enhancement.

In a recent issue of Clinical Endoscopy, Leem et al. ${ }^{6}$ reported the clinical value of CH-EUS in the differential diagnosis of pancreatic and gallbladder masses. While it was not possible to quantitatively measure the degree of enhancement on account of the retrospective study design, the results were sufficient to prove the superiority of $\mathrm{CH}$-EUS over conventional EUS in the differentiation of solid pancreas lesions. The ability of EUS to evaluate both texture and enhancement pattern may improve diagnostic utility, particularly for pancreatic adenocarcinoma. The enhancement pattern alone showed higher sensitivity (82.0\%) and specificity (87.9\%) for ductal 
adenocarcinoma than echogenic pattern alone (sensitivity and specificity of $49.0 \%$ and $93.3 \%$, respectively). When diagnosing neuroendocrine tumors, a combined hyperenhancement and homogeneous pattern on CH-EUS improved sensitivity from $81.1 \%$ to $85.3 \%$, and specificity from $90.9 \%$ to $91.0 \%$, when compared with a hyperenhancement pattern alone. ${ }^{6}$ On the other hand, malignant gallbladder masses did not show a specific enhancement pattern on CH-EUS. For these tumors, the authors used the echogenic and vascular enhancement textures (inhomogeneous or homogeneous) for diagnosis. An inhomogeneous enhancement texture on CH-EUS was the most predictive parameter for diagnosing malignant masses, with a sensitivity of $97.1 \%{ }^{6}$

Recent reports have shown that CH-EUS has a higher diagnostic accuracy for pancreatic cancers than conventional techniques. ${ }^{7-10}$ While the benefits of CH-EUS over conventional EUS are readily apparent, a similar level of discrimination is possible with dynamic MRI or CT. Furthermore, CT or MRI is commonly performed prior to EUS and used as a reference. To determine if CH-EUS is superior to conventional methods, more research that directly compares the utility and accuracy of different modalities is required. Currently, EUS is used to supplement conventional imaging, particularly due to the limitations discussed above. While previous studies have reported superior sensitivity and specificity when using EUS, these reports show significant discrepancies among endoscopists, and often do not report objective findings.

Cancer staging, especially depends on the use of imaging techniques such as CT or MRI for objective characterization and differentiation. Recent improvements in conventional techniques have resulted in higher resolution images, which may widen the gap between these modalities and EUS still further. The role of EUS could change in the future as more research is completed, particularly, if EUS is able to alleviate the need for pathologic diagnosis. At present, EUS serves as a useful adjunct to conventional techniques and may provide additional information; the amount of information that it provides could be further increased through the use of $\mathrm{CH}$ EUS.

$\mathrm{CH}$-EUS has a diverse array of uses including assessment of solid tumors and cystic lesions of the pancreas, characterization of submucosal neoplasms, assessment of biliary neoplasms, and assessment of lymph nodes. Some of the additional benefits and limitations of CH-EUS will be discussed subsequently. CH-EUS has limited utility when attempting to differentiate mass-forming pancreatitis, as it cannot distinguish between normal pancreas parenchymal lesions and focal mass forming inflammatory lesions. Regarding additional benefits, first, $\mathrm{CH}$-EUS does have the ability to visualize small blood vessels within a tumor, if used with ultrasonographic contrast agent. This allows for specific targeting of lesions, while avoiding small blood vessels and necrotic tissue, so that CH-EUS-guided FNA/B may require fewer needle passes. ${ }^{11,12}$ Second, it is difficult to differentiate between mural nodules and mucus clots using conventional EUS method. Contrast-enhanced dynamic CT or MRI can reveal the vascularity of large mural nodules, however has only limited utility for the assessment of small nodules. An advantage of CH-EUS is its ability to identify and characterize relatively small mural nodules and provide a more accurate differentiation by confirming the presence of vascularity. This is invaluable when differentiating between mural nodules and pancreatic cystic lesions. ${ }^{13-15}$ Third, CH-EUS may be applied to cancer staging and post-treatment evaluation. CH-EUS is useful when assessing tumor grade before chemotherapy, and can be used to evaluate the degree of tumor perfusion. The efficacy of successful tumor ablation may be predicted from vascularity changes brought about by chemotherapy, characterized using CH-EUS. The extent of tumor blood flow has been shown to predict chemotherapeutic sensitivity and overall mortality. ${ }^{16}$ Matsui et al. ${ }^{17}$ showed changes in tumor size and vascularity by using a CH-EUS during chemotherapy in patients who have gastric cancer. Highly vascular tumors may be more chemosensitive due to increased drug penetetration. ${ }^{18}$

In conclusion, CH-EUS is a useful and simple technique for the differentiation of pancreas and solid organ tumors. With further technical improvements, it may adequately substitute conventional imaging techniques and allow diagnostic confirmation prior to the decision to proceed with surgery.

\section{Conflicts of Interest}

The author has no financial conflicts of interest.

\section{REFERENCES}

1. Kaufman AR, Sivak MV Jr. Endoscopic ultrasonography in the differential diagnosis of pancreatic disease. Gastrointest Endosc 1989;35:214219.

2. Brugge WR. EUS. Gastrointest Endosc 2013;78:414-420.

3. Ishikawa $\mathrm{H}$, Hirooka $\mathrm{Y}$, Itoh $\mathrm{A}$, et al. A comparison of image quality between tissue harmonic imaging and fundamental imaging with an electronic radial scanning echoendoscope in the diagnosis of pancreatic diseases. Gastrointest Endosc 2003;57:931-936.

4. Matsumoto K, Katanuma A. Novel tissue harmonic imaging clearly visualizes a case of intraductal papillary mucinous neoplasm with mural nodules. JOP 2014;15:274-275.

5. Kitano M, Yamashita Y. New imaging techniques for endoscopic ultrasonography: contrast-enhanced endoscopic ultrasonography. Gastrointest Endosc Clin N Am 2017;27:569-583.

6. Leem G, Chung MJ, Park JY, et al. Clinical value of contrast-enhanced harmonic endoscopic ultrasonography in the differential diagnosis of pancreatic and gallbladder masses. Clin Endosc 2018;51:80-88.

7. Lee TY, Cheon YK, Shim CS. Clinical role of contrast-enhanced harmonic endoscopic ultrasound in differentiating solid lesions of the pan- 
creas: a single-center experience in Korea. Gut Liver 2013;7:599-604.

8. Kitano M, Kudo M, Yamao K, et al. Characterization of small solid tumors in the pancreas: the value of contrast-enhanced harmonic endoscopic ultrasonography. Am J Gastroenterol 2012;107:303-310.

9. Fusaroli P, Spada A, Mancino MG, Caletti G. Contrast harmonic echo-endoscopic ultrasound improves accuracy in diagnosis of solid pancreatic masses. Clin Gastroenterol Hepatol 2010;8:629-634.e1-e2.

10. Napoleon B, Alvarez-Sanchez MV, Gincoul R, et al. Contrast-enhanced harmonic endoscopic ultrasound in solid lesions of the pancreas: results of a pilot study. Endoscopy 2010;42:564-570.

11. Sugimoto $M$, Takagi T, Hikichi T, et al. Conventional versus contrast-enhanced harmonic endoscopic ultrasonography-guided fine-needle aspiration for diagnosis of solid pancreatic lesions: a prospective randomized trial. Pancreatology 2015;15:538-541.

12. Kitano M, Kamata K. Contrast-enhanced harmonic endoscopic ultrasound: future perspectives. Endosc Ultrasound 2016;5:351-354.

13. Zhong N, Zhang L, Takahashi N, et al. Histologic and imaging features of mural nodules in mucinous pancreatic cysts. Clin Gastroenterol
Hepatol 2012;10:192-198, 198.e1-e2.

14. Harima H, Kaino S, Shinoda S, Kawano M, Suenaga S, Sakaida I. Differential diagnosis of benign and malignant branch duct intraductal papillary mucinous neoplasm using contrast-enhanced endoscopic ultrasonography. World J Gastroenterol 2015;21:6252-6260.

15. Ohno E, Hirooka Y, Itoh A, et al. Intraductal papillary mucinous neoplasms of the pancreas: differentiation of malignant and benign tumors by endoscopic ultrasound findings of mural nodules. Ann Surg 2009;249:628-634.

16. Sofuni A, Itoi T, Itokawa F, et al. Usefulness of contrast-enhanced ultrasonography in determining treatment efficacy and outcome after pancreatic cancer chemotherapy. World J Gastroenterol 2008;14:7183-7191.

17. Matsui S, Kudo M, Kitano M, Asakuma Y. Evaluation of the response to chemotherapy in advanced gastric cancer by contrast-enhanced harmonic EUS. Hepatogastroenterology 2015;62:595-598.

18. Yamashita $\mathrm{Y}$, Ueda $\mathrm{K}$, Itonaga $\mathrm{M}$, et al. Tumor vessel depiction with contrast-enhanced endoscopic ultrasonography predicts efficacy of chemotherapy in pancreatic cancer. Pancreas 2013;42:990-995. 